Honam Mathematical J. 35 (2013), No. 3, pp. 507-513

http://dx.doi.org/10.5831/HMJ.2013.35.3.507

\title{
MODULAR TRANSFORMATION FORMULAE FOR GENERALIZED NON-HOLOMORPHIC EISENSTEIN SERIES
}

\author{
Sung-Geun Lim
}

\begin{abstract}
In this paper, we compute transformation formulae for generalized non-holomorphic Eisenstein series.
\end{abstract}

\section{Introduction}

J. Lewittes [3] proved transformation formulae for a large class of Eisenstein series which is defined by

$$
G\left(z, s, r_{1}, r_{2}\right)=\sum_{m, n=-\infty}^{\infty}\left(\left(m+r_{1}\right) z+n+r_{2}\right)^{-s},
$$

where the dash ' means $(m, n) \neq\left(-r_{1},-r_{2}\right), \operatorname{Im} z>0, r_{1}, r_{2}$ are real numbers and $\operatorname{Re} s>2$. However, Lewittes's results were too complicated to deduce desired results. It was B. C. Berndt [1] who has obtained considerably simpler formulae than Lewittes's results. Berndt [2] also proved transformation formulae for a more general class of Eisenstein series than Lewittes's case, which is defined by

$$
G\left(z, s ; r_{1}, r_{2}, h_{1}, h_{2}\right):=\sum_{m, n=-\infty}^{\infty} \frac{e^{2 \pi i\left(m h_{1}+n h_{2}\right)}}{\left(\left(m+r_{1}\right) z+n+r_{2}\right)^{s}},
$$

where the dash ' means $(m, n) \neq\left(-r_{1},-r_{2}\right)$, Im $z>0, r_{1}, r_{2}, h_{1}, h_{2}$ are real and $\operatorname{Re} s>2$.

In this paper, we consider a class of generalized non-holomorphic Eisenstein series. In fact, H. Maass [5] considered non-holomorphic Eisenstein series(when $r=h=(0,0)$ in section 3 ) as an important thing of the theory of non-holomorphic modular forms and determined the Fourier

Received July 3, 2013. Accepted July 13, 2013.

2010 Mathematics Subject Classification. Primary 34A25; Secondary 11M36.

Key words and phrases. Modular transformation; Eisenstein series. 
series of this non-holomorphic Eisenstein series. We obtain modular transformation formulae for generalized non-holomorphic Eisenstein series.

\section{Notations}

If $w$ is a complex, we choose the branch of the argument defined by $-\pi \leq \arg w<\pi$. We write $e(w)$ for $e^{2 \pi i w}$. Let $\lambda$ denote the characteristic function of the integers. In the sequel, $V \tau=V(\tau)=\frac{a \tau+b}{c \tau+d}$ always denotes a modular transformation with $c>0$ for every complex $\tau$. Let $r=\left(r_{1}, r_{2}\right)$ and $h=\left(h_{1}, h_{2}\right)$ denote real vectors, and define the associated vectors $R$ and $H$ by

$$
R=\left(R_{1}, R_{2}\right)=\left(a r_{1}+c r_{2}, b r_{1}+d r_{2}\right)
$$

and

$$
H=\left(H_{1}, H_{2}\right)=\left(d h_{1}-b h_{2},-c h_{1}+a h_{2}\right) .
$$

For real $x, \alpha$ and $\operatorname{Re} s>1$, let

$$
\psi(x, \alpha, s):=\sum_{n+\alpha>0} \frac{e(n x)}{(n+\alpha)^{s}} .
$$

For a real number $x,[x]$ denotes the greatest integer less than or equal to $x$ and $\{x\}:=x-[x]$. Let ${ }_{2} F_{1}(\alpha, \beta ; \gamma ; z)$ be a hypergeometric function defined by

$$
{ }_{2} F_{1}(\alpha, \beta ; \gamma ; z):=\sum_{n=0}^{\infty} \frac{(\alpha)_{n}(\beta)_{n}}{(\gamma)_{n} n !} z^{n},
$$

where $(x)_{n}$ denotes the rising factorial defined by

$$
(x)_{n}:=x(x+1) \cdots(x+n-1) \text { for } n>0,(x)_{0}:=1 .
$$

Euler's integral representation of ${ }_{2} F_{1}$-hypergeometric function says that, for $\operatorname{Re} \gamma>\operatorname{Re} \alpha>0$ and $z \in \mathbb{C} \backslash[1, \infty)$,

$$
{ }_{2} F_{1}(\alpha, \beta ; \gamma ; z)=\frac{\Gamma(\gamma)}{\Gamma(\alpha) \Gamma(\gamma-\alpha)} \int_{0}^{1} t^{\alpha-1}(1-t)^{\gamma-\alpha-1}(1-z t)^{-\beta} d t
$$

Thus we see that $[4,6]$

$$
\frac{1}{\Gamma(\gamma)}{ }_{2} F_{1}(\alpha, \beta ; \gamma ; z)
$$

can be analytically continued to all $\alpha, \beta, \gamma \in \mathbb{C}$ and all $z \in \mathbb{C} \backslash[1, \infty)$. 


\section{Generalized non-holomorphic Eisenstein series}

Let $\mathbb{H}=\{\tau \in \mathbb{C} \mid \operatorname{Im}(\tau)>0\}$, the upper half-plane.

Definition 3.1. Let $\tau \in \mathbb{H}$ and $s, s_{1} \in \mathbb{C}$. For Re $s>2$, the generalized non-holomorphic Eisenstein series $\mathbf{G}\left(\tau, \bar{\tau}, s, s_{1} ; r, h\right)$ is defined to be

$$
\mathbf{G}\left(\tau, \bar{\tau}, s, s_{1} ; r, h\right):=\sum_{m, n=-\infty}^{\infty} \frac{e\left(m h_{1}+n h_{2}\right)}{\left(\left(m+r_{1}\right) \tau+n+r_{2}\right)^{s_{1}}\left(\left(m+r_{1}\right) \bar{\tau}+n+r_{2}\right)^{s-s_{1}}},
$$

where the dash ' means $(m, n) \neq\left(-r_{1},-r_{2}\right)$.

Theorem 3.2. Let $Q=\{\tau \in \mathbb{H} \mid \operatorname{Re} \tau>-d / c\}$ and $\varrho=c\left\{R_{2}\right\}-$ $d\left\{R_{1}\right\}$. Then for $\tau \in Q, s_{1} \in \mathbb{C}$ and $s \in \mathbb{C}$ with $\operatorname{Re} s>2$,

$$
\begin{aligned}
& (c \tau+d)^{-s_{1}}(c \bar{\tau}+d)^{-s+s_{1}} \mathbf{G}\left(V \tau, V \bar{\tau}, s, s_{1} ; r, h\right) \\
& =\mathbf{G}\left(\tau, \bar{\tau}, s, s_{1} ; R, H\right)-2 i \sin (\pi s) \lambda\left(R_{1}\right) e\left(-R_{1} H_{1}\right) \psi\left(-H_{2},-R_{2}, s\right) \\
& \quad+\frac{e\left(-\frac{s}{2}\right)}{\Gamma\left(s_{1}\right) \Gamma\left(s-s_{1}\right)} \mathbf{L}\left(\tau, \bar{\tau}, s, s_{1}, ; R, H\right),
\end{aligned}
$$

where

$$
\begin{aligned}
& \mathbf{L}\left(\tau, \bar{\tau}, s, s_{1} ; R, H\right) \\
& :=\sum_{j=1}^{c} e\left(-H_{1}\left(j+\left[R_{1}\right]-c\right)-H_{2}\left(\left[R_{2}\right]+1+[(j d+\varrho) / c]-d\right)\right) \\
& \cdot \int_{0}^{1} v^{s_{1}-1}(1-v)^{s-s_{1}-1} \int_{C} u^{s-1} \frac{e^{-((c \tau+d) v+(c \bar{\tau}+d)(1-v))\left(j-\left\{R_{1}\right\}\right) u / c}}{e^{-((c \tau+d) v+(c \bar{\tau}+d)(1-v)) u}-e\left(c H_{1}+d H_{2}\right)} \\
& \cdot \frac{e^{\{(j d+\varrho) / c\} u}}{e^{u}-e\left(-H_{2}\right)} d u d v,
\end{aligned}
$$

where $C$ is a loop beginning at $+\infty$, proceeding in the upper half-plane, encircling the origin in the positive direction so that $u=0$ is the only zero of

$$
\left(e^{-((c \tau+d) v+(c \bar{\tau}+d)(1-v)) u}-e\left(c H_{1}+d H_{2}\right)\right)\left(e^{u}-e\left(-H_{2}\right)\right)
$$

lying "inside" the loop, and then returning to $+\infty$ in the lower half plane. Here, we choose the branch of $u^{s}$ with $0<\arg u<2 \pi$.

Proof. For $M=a m+c n, N=b m+d n$,

$$
\begin{aligned}
\left(\left(m+r_{1}\right) V \tau+n+r_{2}\right)^{s} & =\left(\left(m+r_{1}\right) \frac{a \tau+b}{c \tau+d}+n+r_{2}\right)^{s} \\
& =\left(\frac{\left(M+R_{1}\right) \tau+N+R_{2}}{c \tau+d}\right)^{s} .
\end{aligned}
$$


If $m$ and $n$ run over all integers, then $M$ and $N$ run over all integer except for $(M, N)=\left(-R_{1},-R_{2}\right)$ since $a d-b c=1$. Using (3.2), we see that

$\mathbf{G}\left(V \tau, V \bar{\tau}, s, s_{1} ; r, h\right)=\sum_{M, N=-\infty}^{\infty} \frac{e\left(M H_{1}+N H_{2}\right)}{\left(\frac{\left(M+R_{1}\right) \tau+N+R_{2}}{c \tau+d}\right)^{s_{1}}\left(\frac{\left(M+R_{1}\right) \bar{\tau}+N+R_{2}}{c \bar{\tau}+d}\right)^{s-s_{1}}}$.

Thus, by Lemma 1 in [1],

$$
\begin{aligned}
(c \tau+d)^{-s_{1}}(c \bar{\tau}+d)^{-s+s_{1}} \mathbf{G}\left(V \tau, V \bar{\tau}, s, s_{1} ; r, h\right) & \frac{e\left(m H_{1}+n H_{2}\right)}{=} e(-s) \sum_{(m, n) \in I} \frac{e\left(m H_{1}+n H_{2}\right)}{\left(\left(m+R_{1}\right) \tau+n+R_{2}\right)^{s_{1}}\left(\left(m+R_{1}\right) \bar{\tau}+n+R_{2}\right)^{s-s_{1}}} \\
& \quad+\sum_{(m, n) \notin I}^{\prime} \frac{\left(\left(m+R_{1}\right) \tau+n+R_{2}\right)^{s_{1}}\left(\left(m+R_{1}\right) \bar{\tau}+n+R_{2}\right)^{s-s_{1}}}{(3.3)=} \mathbf{G}\left(\tau, \bar{\tau}, s, s_{1} ; R, H\right)+(e(-s)-1) \mathbf{g}\left(\tau, \bar{\tau}, s, s_{1} ; R, H\right),
\end{aligned}
$$

where

$$
\begin{aligned}
& \mathbf{g}\left(\tau, \bar{\tau}, s, s_{1} ; R, H\right) \\
& :=\sum_{(m, n) \in I} \frac{e\left(m H_{1}+n H_{2}\right)}{\left(\left(m+R_{1}\right) \tau+n+R_{2}\right)^{s_{1}}\left(\left(m+R_{1}\right) \bar{\tau}+n+R_{2}\right)^{s-s_{1}}},
\end{aligned}
$$

and

$$
I:=\left\{(m, n) \in \mathbb{Z} \times \mathbb{Z} \mid m+R_{1} \leq 0, d\left(m+R_{1}\right)>c\left(n+R_{2}\right)\right\} .
$$

Replacing $m$ by $-m$ and $n$ by $-n$, and separating the sum for $m=-R_{1}$, we have

$$
\begin{gathered}
\mathbf{g}\left(\tau, \bar{\tau}, s, s_{1} ; R, H\right)=\lambda\left(R_{1}\right) e\left(\frac{s}{2}\right) e\left(-R_{1} H_{1}\right) \psi\left(-H_{2},-R_{2}, s\right) \\
+e\left(\frac{s}{2}\right) \mathbf{h}\left(\tau, \bar{\tau}, s, s_{1} ; R, H\right)
\end{gathered}
$$

where

$$
\begin{aligned}
& \mathbf{h}\left(\tau, \bar{\tau}, s, s_{1} ; R, H\right) \\
& :=\sum_{(m, n) \in J} \frac{e\left(-m H_{1}-n H_{2}\right)}{\left(\left(m-R_{1}\right) \tau+n-R_{2}\right)^{s_{1}}\left(\left(m-R_{1}\right) \bar{\tau}+n-R_{2}\right)^{s-s_{1}}},
\end{aligned}
$$

where

$$
J:=\left\{(m, n) \in \mathbb{Z} \times \mathbb{Z} \mid m-R_{1}>0, d\left(m-R_{1}\right)<c\left(n-R_{2}\right)\right\} .
$$


We now use Euler's integral representation of $\Gamma(s)$ to obtain that, for $\tau \in Q$,

$$
\begin{aligned}
& \Gamma\left(s-s_{1}\right) \Gamma\left(s_{1}\right) \mathbf{h}\left(\tau, \bar{\tau}, s, s_{1} ; R, H\right)=\sum_{(m, n) \in J} e\left(-m H_{1}-n H_{2}\right) \\
& \cdot \int_{0}^{\infty} \int_{0}^{\infty} u^{s_{1}-1} v^{s-s_{1}-1} e^{-\left(m-R_{1}\right)(\tau u+\bar{\tau} v)-\left(n-R_{2}\right)(u+v)} d u d v .
\end{aligned}
$$

Replace indices, $m$ by $m+\left[R_{1}\right]+1$ and $n$ by $n+\left[R_{2}+\left(d\left(m-R_{1}\right) / c\right]+1\right.$ to find that

$$
\begin{aligned}
& \Gamma\left(s-s_{1}\right) \Gamma\left(s_{1}\right) \mathbf{h}\left(\tau, \bar{\tau}, s, s_{1} ; R, H\right) \\
& =\sum_{m, n=0}^{\infty} e\left(-H_{1}\left(m+\left[R_{1}\right]+1\right)-H_{2}\left(n+\left[R_{2}\right]+1+[(\varrho+m d+d) / c]\right)\right) \\
& \cdot \int_{0}^{\infty} \int_{0}^{\infty} u^{s_{1}-1} v^{s-s_{1}-1} e^{-\left(m-\left\{R_{1}\right\}+1\right)(\tau u+\bar{\tau} v)-\left(n-\left\{R_{2}\right\}+1+[(\varrho+m d+d) / c]\right)(u+v)} d u d v .
\end{aligned}
$$

Let $m=q c+j-1,0 \leq q<\infty, 1 \leq j \leq c$. Involving sum of geometric series and manipulation of integral, we find that

$$
\begin{aligned}
& \Gamma\left(s-s_{1}\right) \Gamma\left(s_{1}\right) \mathbf{h}\left(\tau, \bar{\tau}, s, s_{1} ; R, H\right) \\
& =\sum_{j=1}^{c} e\left(-H_{1}\left(j+\left[R_{1}\right]\right)-H_{2}\left(\left[R_{2}\right]+1+[(\varrho+j d) / c]\right)\right) \\
& \cdot \int_{0}^{\infty} \int_{0}^{\infty} u^{s_{1}-1} v^{s-s_{1}-1} e^{-\left(j-\left\{R_{1}\right\}+1\right)(\tau u+\bar{\tau} v)-\left(1-\left\{R_{2}\right\}+[(\varrho+j d) / c]\right)(u+v)} \\
& \sum_{q=0}^{\infty} \sum_{n=0}^{\infty} e^{-q\left(2 \pi i\left(c H_{1}+d H_{2}\right)+(c \tau+d) u+(c \bar{\tau}+d) v\right)-n\left(2 \pi i H_{2}+u+v\right)} d u d v \\
& =\sum_{j=1}^{c} e\left(-H_{1}\left(j+\left[R_{1}\right]-c\right)-H_{2}\left(\left[R_{2}\right]+1+[(\varrho+j d) / c]-d\right)\right) \\
& \cdot \int_{0}^{\infty} \int_{0}^{\infty} u^{s_{1}-1} v^{s-s_{1}-1} \frac{e^{-(c \tau+d)\left(j-\left\{R_{1}\right\}\right) u / c-(c \bar{\tau}+d)\left(j-\left\{R_{1}\right\}\right) v / c}}{e\left(c H_{1}+d H_{2}\right)-e^{-(c \tau+d) u-(c \bar{\tau}+d) v}} \\
& \cdot \frac{e^{\left\{\frac{e+j d}{c}\right\}(u+v)}}{e^{u+v}-e\left(-H_{2}\right)} d u d v \\
& =\sum_{j=1}^{c} e\left(-H_{1}\left(j+\left[R_{1}\right]\right)-H_{2}\left(\left[R_{2}\right]+1+[(\varrho+j d) / c]\right)\right) \\
& \cdot \int_{0}^{1} v^{s_{1}-1}(1-v)^{s-s_{1}-1} \int_{0}^{\infty} u^{s-1} \frac{e^{-((c \tau+d) v+(c \bar{\tau}+d)(1-v))\left(j-\left\{R_{1}\right\}\right) u / c}}{e\left(c H_{1}+d H_{2}\right)-e^{-((c \tau+d) v+(c \bar{\tau}+d)(1-v)) u}} \\
& \cdot \frac{e^{\left\{\frac{\rho+j d}{c}\right\} u}}{e^{u}-e\left(-H_{2}\right)} d u d v \text {. }
\end{aligned}
$$

The inversion of the order of summations and integrations can be justified by absolute convergence of the original series for $\operatorname{Re} s>2$. This 
last integrals from 0 to $\infty$ can be converted to loop integrals on $C$ by the same manner in [1]. Hence, for $\operatorname{Re} s>2$ and $\tau \in Q$, it follows that

$$
\begin{aligned}
& \Gamma\left(s-s_{1}\right) \Gamma\left(s_{1}\right) \mathbf{h}\left(\tau, \bar{\tau}, s, s_{1} ; R, H\right) \\
& =-\frac{1}{e(s)-1} \sum_{j=1}^{c} e\left(-H_{1}\left(j+\left[R_{1}\right]\right)-H_{2}\left(\left[R_{2}\right]+1+[(\varrho+j d) / c]\right)\right) \\
& \quad \cdot \int_{0}^{1} v^{s_{1}-1}(1-v)^{s-s_{1}-1} \int_{C} u^{s-1} \frac{e^{-((c \tau+d) v+(c \bar{\tau}+d)(1-v))\left(j-\left\{R_{1}\right\}\right) u / c}}{e\left(c H_{1}+d H_{2}\right)-e^{-((c \tau+d) v+(c \bar{\tau}+d)(1-v)) u}} \\
& \cdot \frac{e^{\left\{\frac{\varrho+j d}{c}\right\} u}}{e^{u}-e\left(-H_{2}\right)} d u d v .
\end{aligned}
$$

Putting (3.5) into (3.4) and then (3.4) into (3.3), the proof is done.

We see that each loop integrals in (3.5) converges uniformly on any compact set of the $s$-plane. Thus, using the analytic continuation of ${ }_{2} F_{1}(\alpha, \beta ; \gamma ; z) / \Gamma(\gamma)$ and $(2.2)$ and applying the same manner in [4], p. 239 , we find that

$$
\frac{1}{\Gamma\left(s_{1}\right) \Gamma\left(s-s_{1}\right)} \mathbf{L}\left(\tau, \bar{\tau}, s, s_{1}, ; R, H\right)
$$

can be analytically continued to all values of $s_{1}$ and $s$. Now if $s$ is an integer, then integrals may be computed using the residue theorem. We use the generating function

$$
\frac{t e^{x t}}{e^{t}-1}=\sum_{n=0}^{\infty} B_{n}(x) \frac{t^{n}}{n !}(|t|<2 \pi)
$$

for Bernoulli polynomials $B_{n}(x), n \geq 0$. The $n$-th Bernoulli number $B_{n}$, $n \geq 0$, is defined by $B_{n}=B_{n}(0)$. Put $\bar{B}_{n}(x)=B_{n}(\{x\}), n \geq 0$. Let $s=-N$ for an integer $N$. By the same manner in [1], we find that

$$
\begin{aligned}
& \int_{C} u^{s-1} \frac{e^{-((c \tau+d) v+(c \bar{\tau}+d)(1-v))\left(j-\left\{R_{1}\right\}\right) u / c}}{e^{-((c \tau+d) v+(c \bar{\tau}+d)(1-v)) u}-1} \frac{e^{\left\{\frac{\varrho+j d}{c}\right\} u}}{e^{u}-1} d u \\
& =2 \pi i \sum_{k=0}^{N+2} \frac{B_{k}\left(\frac{j-\left\{R_{1}\right\}}{c}\right) \bar{B}_{N+2-k}\left(\frac{\varrho+j d}{c}\right)}{k !(N+2-k) !}(-c v(\tau-\bar{\tau})-c \bar{\tau}-d)^{k-1} .
\end{aligned}
$$

Thus, replacing $v$ by $1-v$, we obtain that

$$
\begin{aligned}
\int_{0}^{1} v^{s_{1}-1}(1-v)^{-N-s_{1}-1} \int_{C} u^{-N-1} \frac{e^{-((c \tau+d) v+(c \bar{\tau}+d)(1-v))\left(j-\left\{R_{1}\right\}\right) u / c}}{e^{-((c \tau+d) v+(c \bar{\tau}+d)(1-v)) u}-1} \\
\cdot \frac{e^{\left\{\frac{e+j d}{c}\right\} u}}{e^{u}-1} d u d v
\end{aligned}
$$




$$
\begin{aligned}
= & 2 \pi i \sum_{k=0}^{N+2} \frac{B_{k}\left(\frac{j-\left\{R_{1}\right\}}{c}\right) \bar{B}_{N+2-k}\left(\frac{\varrho+j d}{c}\right)}{k !(N+2-k) !}(-c \tau-d)^{k-1} \\
& \cdot \int_{0}^{1}(1-v)^{s_{1}-1} v^{-N-s_{1}-1}\left(1-\frac{c(\tau-\bar{\tau})}{c \tau+d} v\right)^{k-1} d v \\
= & 2 \pi i \frac{\Gamma\left(s_{1}\right) \Gamma\left(-N-s_{1}\right)}{\Gamma(-N)} \sum_{k=0}^{N+2} \frac{B_{k}\left(\frac{j-\left\{R_{1}\right\}}{c}\right) \bar{B}_{N+2-k}\left(\frac{\varrho+j d}{c}\right)}{k !(N+2-k) !}(-c \tau-d)^{k-1} \\
& \cdot{ }_{2} F_{1}\left(-N-s_{1}, 1-k ;-N ; \frac{c(\tau-\bar{\tau})}{c \tau+d}\right) .
\end{aligned}
$$

Acknowledgement. The author thanks B. C. Berndt for his helpful suggestions for this work.

\section{References}

[1] B. C. Berndt, Generalized Eisenstein series and modified Dedekind sums, J. Reine. Angew. Math. 272 (1975), 182-193.

[2] B. C. Berndt, Modular transformations and generalizations of several formulae of Ramanujan, The Rocky mountain J. Math. 7(1) (1977), 147-189.

[3] Joseph Lewittes, Analytic continuation of Eisenstein series, Trans. Amer. Math. Soc. 171 (1972), 469-490.

[4] N. N. Lebedev, Special functions and their applications, Dover Publications, Inc., New York, 1972.

[5] H. Maass, Modular Functions of One Complex Variable, Tata Institute, Bombay, 1964.

[6] E. C. Titchmarsh, Theory of functions, Oxford University Press, 1952.

\section{Sung-Geun Lim}

Department of Mathematics, Mokwon University,

Mokwon Gil 21, Seo-gu, Daejeon, 302-318, Republic of Korea.

E-mail: sglimj@mokwon.ac.kr 